\title{
The Cavity Image Denoising Method Combined Median Filter with Adaptive Filter
}

\author{
Shan Huang, Cheng $\mathrm{Xu}^{*}$ and Yaoke Wen \\ School of Mechanical Engineering, NUST, Nanjing 210094, China \\ *Corresponding author
}

\begin{abstract}
Processing the wound bullet's cavity image has an important value on studying the temporary cavity's formation, evolution and quantitative evaluation of its size. But it is difficult to assure the quality of the image, so the boundary can not be distinguished. This paper proposes a hybrid filter method combined with median filtering and adaptive filtering. Comparing with the average filtering, median filtering and adaptive filtering, the hybrid filter method can constrain and reduce noise effectively, and it can protect the outline's details. According to the calculation by filtering objective quality evaluation methods of MSE, MAE, NMSE, NMAE, the hybrid filtering method is superior to other conventional methods.
\end{abstract}

Keywords-temporary cavity; gelatin; high-speed photography; filtering; evaluation

\section{INTRODUCTION}

Temporary cavity is a rapidly changing physical phenomenon when the high-speed projectile (bullet, chip etc.) penetrating the tissue. Because of the local stress, the tissue is sheared and expanded along the crack. It is an important cause of severely injuring the tissues and organs[1]. The wound bullet's cavity image contains rich information of projectile motion in the target, its features can directly reflect the trajectory's damage degree and range. It has important value in the research of temporary cavity's evolution , and important guiding significance to the design of the ammunition and the improvement of medical technology at the same time[2]. But the image of temporary cavity in gelatin is often influenced by various kinds of noise. It has some shortcomings, such as the low signal-to-noise ratio, the unconspicuous gray difference between the target and background. According to the present knowledge and practice of wound ballistics, it will influence the accurate judgments on the degree and extent of the damage when the temporary cavity's weak edge can't be detected automatically. Many theoretical and experimental studies in trauma ballistics have been carried out and some achievements have been acquired in damage mechanism and trauma characteristics caused by different projectiles[3-5], but the research is reported rarely about the application of image processing technology to temporary cavity's image[6-8]. In order to effectively filter noise in the wound bullet cavity image , offer the basis for subsequent contour extraction and improve measurement precision of the cavity's volume, this text comparative analysis and objective evaluation of the effectiveness in processing image are made in different methods of reducing noise , including the average filtering, median filtering, adaptive filtering and the hybrid filter combined with median filtering and adaptive filtering.

\section{THE COMMON FILTERING ALgORITHM}

The average filtering algorithm which is also called neighborhood average method is a typical linear filter technology, and its basic idea is to make use of several adjacent pixels' gray scale average instead of each pixel gray value. The basic average filtering is the most simple average filter, defining wnd expressing center pixel(i,j), $m \times n$ expressing size of rectangular filter window (for example $3 \times 3$ window). Arithmetic average filtering process is calculating the interferential center pixel's export gray value $f(i, j)$ by the average of pixel in filter window defined as wnd That is:

$$
\begin{gathered}
\text { wnd }=\left(\begin{array}{lcr}
g(i-1, j-1) & g(i, j-1) & g(i+1, j-1) \\
g(i-1, j) & g(i, j) & g(i+1, j) \\
g(i-1, j+1) & g(i, j+1) & g(i+1, j+1)
\end{array}\right) \\
f(i, j)=\text { mean }\{w n d\}=\frac{1}{m n} \sum_{k=1}^{m n} w n d(k)
\end{gathered}
$$

In the above formula, mean expresses averaging of the elements in $w n d$, and $w n d(k)$ is the average for the pixel gray value in the filter window. Basic average filtering simply smoothes the local change of image, reducing noise but blurred images at the same time, and the effects of pulse noise will be spread to the surrounding pixels in the process. This kind of filter does not use the correlation between pixels and location information of the pixels.

The median filtering is a common nonlinear smoothing filter algorithm, this method can protect the image detail and filter the impulse noise effectively at the same time, therefore it has been widely used in the field of image processing. The main principle of basic median filter is that a neighborhood around the pixel at the center should be determined firstly, which is a square neighborhood generally; And then the gray value of each pixel neighborhood is ordered, the median will be selected as a new value of the center pixel gray value, the neighborhood here is often called the window. When the window moves up and down or so, the image can be well smoothed by median 
filtering algorithm. Its algorithms express is used by the median gray value to replace the pixel value:

$$
f(i, j)=\operatorname{med}\{w n d\}
$$

In the formula (3), mean expresses mid-value operation, $f(i, j)$ expresses the filter's output, $g(i, j)$ expresses the pixel which isn't filtered, wnd expresses the filtering window. The median filter is applied to the two-dimensional image processing, and has good inhibition for the pulse signal, but the filtering ability is poor for the gaussian noise. Because the characteristics of gaussian noise is dense, its influence scope is extremely broad, even spread to all points of the whole image. At the same time, the influence on the spread point is small , much smaller than the pulse noise.

The adaptive filter is a good filtering method developed in recent years, which is based on wiener linear filtering. Because of its strong adaptability and good filtering performance, it has been widely used in engineering practice. Adaptive filtering is a restore method which make the mean square error minimize between the original image and the recovery image, its principle is as follows. First of all, estimates the pixels of the local mean and variance:

$$
\begin{gathered}
\mu=\frac{1}{M \cdot N} \sum_{n_{1}, n_{2} \in \eta} a\left(n_{1}, n_{2}\right) \\
\sigma^{2}=\frac{1}{M \cdot N} \sum_{n_{1}, n_{2} \in \eta} a^{2}\left(n_{1}, n_{2}\right)-\mu^{2}
\end{gathered}
$$

In the formula, ${ }^{\eta}$ is the neighbourhood of the each pixel which size is $\mathrm{M} \times \mathrm{N}$.

Then, Winner2 filter is used to estimate its grey value for each pixel .

$$
b\left(n_{1}, n_{2}\right)=\mu+\frac{\sigma^{2}-v^{2}}{\sigma^{2}}\left(a\left(n_{1}, n_{2}\right)-\mu\right)
$$

In the formula, $v^{2}$ is the noise variance in a whole image.

The self-adaptive filter adjusts the output of the filter based on the image's local variance, when the local variance is big, the smoothing effect of filter is small, otherwise the filter's smoothing effect is strong.Winner2 filter can self-adaptively adjust the filter parameters according to the output. It usually has better selectivity than linear filter, and can retain the image's edge details and high frequency information. It can remove gaussian noise obviously, but the effect of filtering the impulse noise is normal.

The median filter can effectively suppress gaussian noise, but the image detail is fuzzy; Median filtering can filter impulse noise in the images, and can keep the image edge well; Self-adaptive filter can remove the gaussian noise better. Here is the comparison of several common filtering methods as shown in table 1.

TABLE I. CONTRAST BETWEEN COMMON FILTERING METHODS

\begin{tabular}{|c|c|c|c|}
\hline $\begin{array}{c}\text { Filtering } \\
\text { method }\end{array}$ & $\begin{array}{c}\text { Restrain } \\
\text { gaussian noise }\end{array}$ & $\begin{array}{c}\text { Restrain } \\
\text { impulse +noise }\end{array}$ & $\begin{array}{c}\text { Protect edge } \\
\text { detail }\end{array}$ \\
\hline $\begin{array}{c}\text { average } \\
\text { filtering }\end{array}$ & well & Worse & Worse \\
\hline median filter & common & well & well \\
\hline $\begin{array}{c}\text { adaptive } \\
\text { filtering }\end{array}$ & well & common & well \\
\hline
\end{tabular}

\section{THE FILTERING ALGORITHM COMBINED WITH THE MEDIAN FILTERING AND ADAPTIVE}

By analyzing the characteristics of the temporary cavity image in the gelatin, it contains two mixed noise of gaussian and impulse. In order to effectively filter the mixed noise and keep the contour information, the selection of filter algorithm is analyzed in the following. 1. Using average filtering can remove the gaussian noise in the image, but the details of the cavity profile becomes vague;2. It's not good to use median filtering for the gaussian noise. Because the gaussian noise is random distribution on the whole image, and it's not the maximum or minimum points, so the median filter can't treat it as a noise to filter out;3. if only using adaptive filter, it is not good for filtering impulsive noise in image .Because the adaptive filter method cannot accurately identify the noise target, and it will treat the gaussian and impulse noise as a mixture target to denoising processing, so it is circumscribed for the gaussian noise removal.To sum up, the method combined with the median filtering and adaptive filtering can effectively remove the impulse and gaussian noise, and also can protect the contour details in the image, and it's good for the subsequent contour extraction. If firstly use adaptive filter for image denoising, and then use median filtering to denoise, the gaussian noise in the image is firstly gotten rid of well. But at the same time, the impulse noise's grey value will flatten out in the images after removing the gaussian noise. The median filtering, relying on the image gray level to determine the maximum and minimum values of noise points, will be hard to test these impulse noise points in the image. So the image is processed by the median filter firstly and it only contain the gaussian noise, then gaussian noise is removed using adaptive filter in the image lastly.

The subjective evaluation of image quality is made by the human observation directly, the subjective feeling and the statistical result according to certain standards of rules or image samples[9]. The subjective assessment generally adopts comparative observation method by comparing the results image with the original image, results image and noise image, different methods to deal with the result image for evaluation. Objective evaluation method mainly adopts the error evaluation method, namely the noise filtering effect of image is evaluated by calculation of the errors between the filtered results and noiseless image. The original image signal and image signal after filtering are expressed as A and B, respectively, and the length and width of the image as $\mathrm{M}$ and $\mathrm{N},(i, j)$ for the pixel coordinates. There are four kinds of commonly used methods: 1 . 
The mean square error MSE; 2. The mean absolute error MAE; 3 . The normalized mean square error NMSE; 4 . The normalized mean absolute error NMAE. Several widely used kinds of the objective evaluation methods for image noise reduction quality are introduced as follows:

Mean Square Error(MSE)

$$
\operatorname{MSE}(A, B)=\frac{1}{M \times N} \sum_{i=1}^{M} \sum_{j=1}^{N}[A(i, j)-B(i, j)]^{2}
$$

Mean Absolute Error(MAE)

$$
\operatorname{MAE}(A, B)=\frac{1}{M \times N} \sum_{i=1}^{M} \sum_{j=1}^{N}|A(i, j)-B(i, j)|
$$

Normalized Mean Square Error(NMSE)

$$
\operatorname{NMSE}(A, B)=\frac{\sum_{i=1}^{M} \sum_{j=1}^{N}[A(i, j)-B(i, j)]^{2}}{\sum_{i=1}^{M} \sum_{j=1}^{N}[A(i, j)]^{2}}
$$

Normalized Mean Absolute Error(NMAE)

$$
\operatorname{NMAE}(A, B)=\frac{\sum_{i=1}^{M} \sum_{j=1}^{N}|A(i, j)-B(i, j)|}{\sum_{i=1}^{M} \sum_{j=1}^{N}|A(i, j)|}
$$

There are advantages and disadvantages in the subjective and objective evaluation method for the image quality. The evaluation principle remains to be further perfected for the latter. While the subjective methods are greatly influenced by the observer and the observation conditions, so the evaluation of noise filtering effect commonly use the method combined with objective and subjective evaluation.

In this chapter, mixed noise is added in gelatin target cavity image. Filtering experiment is carried out in the methods of average filter algorithm, median filtering algorithm, adaptive filtering algorithm and this hybrid algorithm. First, choose a good quality gelatin cavity image of photography by visual measuring system, and the image quality assessment parameters are given by the comparison and analysis, the treatment effect is shown in Figure 1.

From Figure 1, it can be concluded that the average filtering algorithm makes the image blur and the image detail can't be distinguished, the filtering effect is the worst; The median filter can filter the impulse noise in the image, but its smoothing effect on the flat and buffered gray regional are not better than the hybrid algorithm in this paper in the background and foreground, and it can not filter out the gaussian noise well; The adaptive filter can filter out the gaussian noise in image, but it can be seen clearly for the image that there is some residual impulse noise, and the filtering effect isn't better than the median filtering. The denoising effect of using composite algorithm is better than single median filtering and adaptive filtering. Comparing with the single median filtering and adaptive filtering, the denoising effect of composite algorithm is more thoroughly, and it can effectively filter out the gaussian noise and impulse noise, and has better ability to retain the image details. Contrastive analysis of the filter quality of the cavity image is made using four kinds of objective evaluation methods, as illustrated in table 2. Compared with the median filtering algorithm which is similar to the subjective assessment, all the errors of the filtering algorithm in this paper are the minimum, and it obtains the best filtering result from the angle of error statistics analysis.
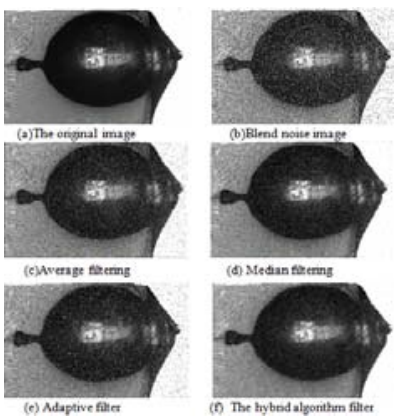

FIGURE I. THE COMPARISON RESULTS OF CAVITY IMAGE'S FILTERING EFFECT

TABLE II. THE OBJECTIVE QUALITY EVALUATION ABOUT THE CAVITY IMAGE FILTERING WITHIN GELATIN TARGETS

\begin{tabular}{c|c|c|c|c}
\hline $\begin{array}{c}\text { Evaluation } \\
\text { methodolog } \\
\text { y }\end{array}$ & $\begin{array}{c}\text { The } \\
\text { average } \\
\text { filtering }\end{array}$ & $\begin{array}{c}\text { The } \\
\text { median } \\
\text { filter }\end{array}$ & $\begin{array}{c}\text { The } \\
\text { adaptive } \\
\text { filtering }\end{array}$ & $\begin{array}{c}\text { The } \\
\text { hybrid } \\
\text { algorith } \\
\text { m filter }\end{array}$ \\
\hline NMSE & $\begin{array}{c}9.6422 \times 1 \\
04\end{array}$ & $3.3243 \times 104$ & $1.1833 \times 105$ & $\begin{array}{c}2.7405 \times 1 \\
04\end{array}$ \\
\hline NMAE & $\begin{array}{c}9.6959 \times 1 \\
04\end{array}$ & $3.4263 \times 104$ & $4.3545 \times 104$ & $\begin{array}{c}3.2591 \times 1 \\
04\end{array}$ \\
\hline MSE & 0.2342 & 0.0044 & 0.0122 & 0.0036 \\
\hline MAE & 0.3764 & 0.0510 & 0.0652 & 0.0482 \\
\hline
\end{tabular}

\section{CONCLUSIONS}

To solve the problem that the temporary cavity image's boundary can't be clearly identified, the composite filtering algorithm is proposed combined with the median filtering and adaptive filtering. And the results of new proposed algorithm are compared with common filtering methods, such as average filtering, median filtering and adaptive filtering. In addition, the filtering effectiveness is evaluated by subjective and objective error statistics. The comparative analysis and evaluation proved that the composite filtering method can effectively restrain and filter out the noise of the image and preserve the detail of contour line. It laid a solid foundation for extracting the contour line and measuring the cavity's volume.

\section{REFERENCES}

[1] Yin-qiu LIU, Zheng-guo WANG, Yu-yuan MA etal. Wound ballistics[M]. Beijing: People’s Medical Press,1991.(in Chinese) 
[2] HE Cheng WANG Tao Zhen-qiang LIAO etal. Research on edge detection of wound ballistics cavity image[J].Computer Engineering and Design,2011,32(1):248-250.

[3] GUO Kai. Acquirement Method of Damage Parameter of Bullet Penetrating Gelatin Target[J].Journal of Ballistics,2014,26(3):87-91.

[4] SHEN Biao, WANG Tao, Zhen-qiang LIAO etal. Wound Ballistics 3D Reconstruction Based on Matlab [J].Journal of Projectiles, Rockets, Missiles and Guidance, 2011,31(1):140-142.

[5] SHEN Jie, WANG Tao, Zhen-qiang LIAO etal. The Processing Technique of Sequential Ballistic Wound Cavity Images Based on Gelatin Experiments[J].Journal of Projectiles, Rockets, Missiles and Guidance,2013,33(2):133-136.

[6] Chuan-zhong WANG, SHI Jun, Zhen-qiang LIAO .Level Set-based Segmentation of the Transient Cavity of Ballistic Wound in X-ray Image[J].JOURNAL OF TEST AND MEASUREMENT TECHNOLOGY,2011, 25(1): 82-86.

[7] LIN Yong, Jing-jin WANG, SONG Zheng etal. Gelatin cavity 3-D reconstruction from flash X-ray images of wound ballistics[J].J Tsinghua Univ (Sci\&Tech),2002,42(12):1576-1578.

[8] Jing-jin WANG, YUAN Jie, Ya-qiang LIU etal. Network based multi-channel digital flash X-ray imaging system[J].Nuclear Electronics \& Detection Technology,2003,23(1):1-3.

[9] Teng-fei WU. Research and Application on Key Algorithms of Image Processing in Vision Measurement[D].Tianjin : Master's degree thesis of Tianjin University,2010. 